\title{
Estimation of the transmission dynamics of Theileria equi and Babesia caballi in horses
}

\author{
S. R. RÜEGG ${ }^{1} \dagger$, D. HEINZMANN ${ }^{1,2} \dagger, A . D . B^{2}$ ARBOUR ${ }^{2}$ and P. R. TORGERSON ${ }^{1 *}$ \\ ${ }^{1}$ Institute of Parasitology, University of Zürich, 8057 Zürich, Switzerland \\ ${ }^{2}$ Institute of Mathematics, University of Zürich, 8057 Zürich, Switzerland
}

(Received 11 Fuly 2007; revised 13 November and 13 December 2007; accepted 17 December 2007; first published online 27 February 2008)

\author{
SUMMARY
}

For the evaluation of the epidemiology of Theileria equi and Babesia caballi in a herd of 510 horses in SW Mongolia, several mathematical models of the transmission dynamics were constructed. Because the field data contain information on the presence of the parasite (determined by PCR) and the presence of antibodies (determined by IFAT), the models cater for maternal protection with antibodies, susceptible animals, infected animals and animals which have eliminated the parasite and also allow for age-dependent infection in susceptible animals. Maximum likelihood estimation procedures were used to estimate the model parameters and a Monte Carlo approach was applied to select the best fitting model. Overall, the results are in line with previous experimental work, and add evidence that the epidemiology of T. equi differs from that of Babesia spp. The presented modelling approach provides a useful tool for the investigation of some vector-borne diseases and the applied model selection procedure avoids asymptotical assumptions that may not be adequate for the analysis of epidemiological field data.

Key words: Theileria equi, Babesia caballi, horses, tick-borne disease, epidemiology, Mongolia, mathematical modelling, Monte Carlo methods.

\section{INTRODUCTION}

Equine piroplasmoses are caused by two intraerythrocytic protozoa, Theileria equi and Babesia caballi. Both are transmitted by ixodid ticks (Friedhoff, 1988). Clinical signs of infection may vary from asymptomatic to acute fever, anaemia and dyspnoea, and even death (reviewed in Schein, 1988). Chronically infected horses represent a reservoir infecting ticks, which subsequently transmit the parasites to other equids. Piroplasms can be detected directly by microscopical examination of Giemsa-stained thin blood smears or by polymerase chain reaction (PCR) (Bruning, 1996), and for indirect diagnosis, the immunofluorescence antibody test (IFAT) is the most widely used technique (Gummow et al. 1996; Avarzed et al. 1997; Heuchert et al. 1999).

The epidemiology of equine piroplasmoses has been investigated in various studies (Mahoney, 1969; Mahoney and Ross, 1972; Ross and Mahoney, 1974; Smith, 1983; Dallwitz et al. 1987; Medley et al. 1993). In 2004, a study was conducted in a domestic horse population in south-western Mongolia (Rüegg

* Corresponding author: Institute of Parasitology, University of Zürich, 8057 Zürich, Switzerland. Tel: +414463585 35. Fax: +414463589 07. E-mail: paul. torgerson@access.unizh.ch

$\dagger$ These authors contributed equally to the manuscript. et al. 2007). PCR results indicated a $T$. equi prevalence of $66 \cdot 5 \%(95 \% \mathrm{CI}: 62 \cdot 2-70 \cdot 7)$ and the IFA test demonstrated that $78 \cdot 8 \%(95 \% \mathrm{CI}: 74 \cdot 9-82 \cdot 3)$ of animals had seroconverted to $T$. equi. The corresponding values for B. caballi were $19 \cdot 1 \%$ (95\% CI : $15 \cdot 7-22 \cdot 8)$ and $65 \cdot 7 \%(95 \% \mathrm{CI}: 61 \cdot 4-69.9)$ respectively. To investigate the impact of age, herd affiliation, sex, date of sample collection and tick abundance on the PCR and IFAT prevalences, a generalized linear model (GLM) and a generalized additive model (GAM) were used. In both models, sex and age were the only two significant explanatory variables (Rüegg et al. 2007). Despite being useful for detection of risk factors and testing hypotheses about relationships of explanatory variables, GLMs and GAMs are in general not adequate to gain insight into the transmission process. Hence, in this work, a mathematical model describing the transmission dynamics is applied which yields a biological interpretation of the prevalence in horses in terms of transmission parameters. For Theileria such a mathematical transmission model has been presented based on the epidemiology of east coast fever (ECF, T. parva) in cattle (Medley et al. 1993). A model for Babesia in cattle has existed since the 1960s (Mahoney, 1969) and has been extended in various versions (Mahoney and Ross, 1972; Ross and Mahoney, 1974; Smith, 1983). These models have been used to simulate the prevalence of piroplasms in cattle and to evaluate strategic interventions in the 
epidemiological cycle. Dallwitz et al. (1987) suggested that because Theileria and Babesia have primarily quantitative rather than qualitative differences in their transmission dynamics, a single model should be able to summarize the biological particularities of both. Therefore, in the present work a series of nested models for different transmission scenarios is presented. Their application to the field data from Mongolia provides insight into the transmission process as opposed to the purely descriptive tools of GLMs and GAMs (Rüegg et al. 2007). An exhaustive Monte Carlo simulation study is conducted to select the best fitting model for both parasites.

\section{MATERIALS AND METHODS}

All procedures were performed using the statistical software R 2.4.0 (R Development Core Team, 2006). The Monte Carlo simulation study was conducted on the high performance computing cluster system 'Matterhorn' of the University of Zürich, providing a floating point performance of approximately 2.9 TFlops with 10 TByte memory. The data file and the code for the models are available online as supplementary data.

\section{Two compartment model}

In a first approach, the dynamics of parasite acquisition and elimination are described with a simple two-compartment model. The population consists of a group of non-infected animals $(S)$ and a group of infected animals $(I)$. The model describes the agedependent dynamics between the two groups. A proportion of the horses $(I(0))$ is infected at birth. Susceptible (non-infected) animals transfer to the infected group with an age-independent acquisition rate $\beta$. Hence $\beta$ can be considered as the prevailing infection pressure per time unit and population. With an age-independent rate $\mu$, infected animals lose all parasites and return into the susceptible group. The model is graphically represented in Fig. 1 and the corresponding ordinary differential equation (ODE) is given in equation (1). A constant population size is assumed, i.e. there is no immigration or emigration. Integrating ODE (1) over the time-interval $[0, t]$ yields the age-dependent prevalence equation (2). Note that $I(0)$ is the initial proportion of infected animals.

$\frac{d I}{d t}=\beta(1-I)-\mu I$

$I(t)=\frac{\beta}{\beta+\mu}+\left(I(0)-\frac{\beta}{\beta+\mu}\right) e^{-(\beta+\mu) t}$,

where $I(t)=$ proportion of horses in the infected group at age $t$;

$\beta=$ rate of acquisition of infection;

$\mu=$ rate of loss of infection.

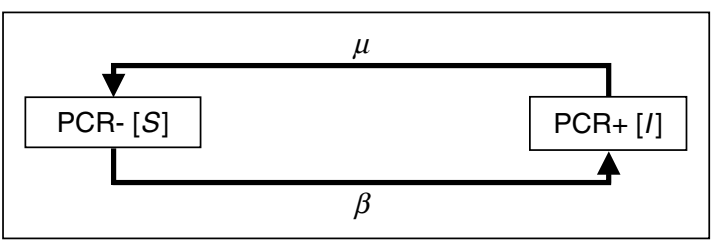

Fig. 1. Graphical representation of model 10. The model considers an age-independent acquisition rate $\beta$ and an elimination rate $\mu$. Two compartments represent the susceptible $(\mathrm{PCR}-)$ and infected $(\mathrm{PCR}+)$ animals in the population. Individuals move between the two compartments at the end of each age interval.

Equation (2) will be denoted as model 10. A complete list of all models used in this paper is given in Table 3. Model 10 is a logical extension of the model presented by Mahoney (1969), which was adapted from malaria to the case of bovine babesiosis under the assumption of endemic stability. It explained the proportion of infected animals $(I(t))$ in terms of the recovery rate $(r)$, the inoculation $(h)$ and the age $(t)$ of the individual animal:

$I(t)=\frac{h}{r}\left(1-e^{-r t}\right)$.

Mahoney's model (3) needs to be restricted such that $r \geqslant h$ to obtain an asymptotic antigen-prevalence not exceeding 1 . In contrast, the present model is self-restricting due to the term $\frac{\beta}{\beta+\mu}$. Since Mahoney and coworkers assumed that there are no infections present at birth, i.e. $I(t=0)=0, \beta$ corresponds to their inoculation rate $h$ and $\beta+\mu$ to their recovery rate $r$.

\section{Four compartment model}

Model 10 oversimplifies the disease transmission because one would expect that immunity of an individual animal influences successful establishment of an infection. Therefore, the two-compartment model is expanded by including the immune status information of each animal (model 20, see equation (5)). This additional information is obtained using an immunofluorescence antibody test (IFAT) and leads to a new subdivision of the population into 4 compartments as shown in Table 1. The dynamics between these compartments correspond to the events during an infection under endemic conditions and are graphically represented in Fig. 2. They can be explained as follows. A proportion of animals is born with maternal antibodies and is thus IFAT positive and PCR negative (IFAT +/PCR - ). They lose their maternal antibodies, i.e. become IFAT $-/ \mathrm{PCR}-$, or they are infected and thus become IFAT $+/ \mathrm{PCR}+$. IFAT $-/ \mathrm{PCR}-$ individuals acquire the parasite $(\mathrm{IFAT}-/ \mathrm{PCR}+)$ before generating antibodies against the pathogen $(\mathrm{IFAT}+/ \mathrm{PCR}+)$. Because this seroconversion requires a relatively short time lag of a few weeks, the differentiation of these two states 
Table 1. PCR and IFAT status of animals in the four-compartment models

\begin{tabular}{lll}
\hline \hline & $\begin{array}{l}\text { PCR } \\
\text { positive } \\
\left(x_{i}=1\right)\end{array}$ & $\begin{array}{l}\text { PCR } \\
\text { negative } \\
\left(x_{i}=0\right)\end{array}$ \\
\hline IFAT positive $\left(\mathrm{y}_{\mathrm{i}}=1\right)$ & $\mathrm{I}$ & $\begin{array}{l}\mathrm{M} \text { : maternal antibodies } \\
\mathrm{R}: \text { acquired antibodies }\end{array}$ \\
IFAT negative $\left(\mathrm{y}_{\mathrm{i}}=0\right)$ & $\mathrm{I}$ & $\mathrm{S}$ \\
\hline \hline
\end{tabular}

is considered to be negligible in the model. Once infected, the immune reaction is either successful and eliminates the parasite $(\mathrm{IFAT}+/ \mathrm{PCR}-)$, or the animal remains positive $(\mathrm{IFAT}+/ \mathrm{PCR}+)$ due to an unsuccessful elimination. If the parasites are eliminated, the antibodies are eventually lost (IFAT -/ $\mathrm{PCR}-$ ) or the animal is re-infected (IFAT +/ PCR +). For each time-interval, each animal in the population is in exactly one of the defined states. At the end of an interval, each animal either moves to a new state or remains in the same state for another interval. The animals move from one state to the next according to transition rates which depend only on the current state (they do not take any previous history into account). These rates are represented in Fig. 2 and their biological interpretation is explained in Table 2. Note that the transition rates in the model do not change over time and that all parameters have a corresponding epidemiological meaning: $\beta$ is the infection rate for seronegative individuals, whereas $e$ and $f$ represent the infection rates for animals with acquired immunity and passive (maternal) immunity, respectively. The elimination rate of the parasite corresponds to $\mu$, and $d$ is the rate at which acquired antibodies are lost after infection, whereas $a$ represents the constitutive loss of maternal antibodies. The whole dynamical process can be written as a system of ODEs in matrix form as

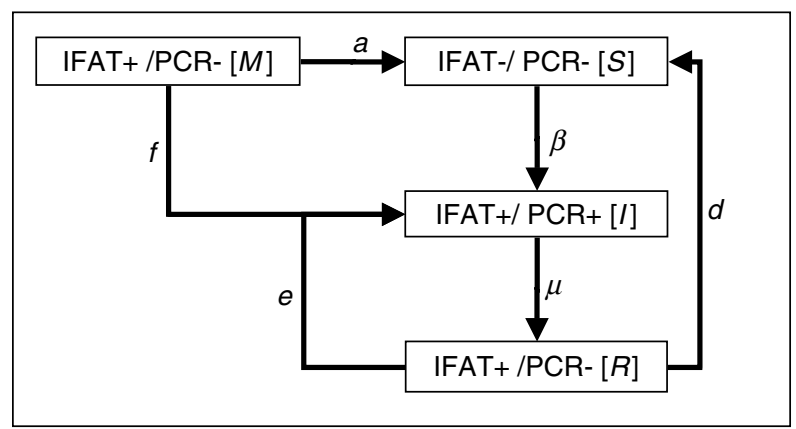

Fig. 2. Graphical representation of model 20. Four compartments represent the maternally protected subpopulation $(M)$, the populations of susceptible $(S)$, infected $(I)$ and immune $(R)$ animals. At the end of each age-interval animals move between the compartments with age-independent rates. The rates $\beta, e$ and $f$ correspond respectively to the acquisition rates without humoral protection of the host, with acquired immunity and with passive maternal protection. The parasites are eliminated with rate $\mu$, and $a$ and $d$ are the respective rates at which maternal and acquired antibodies are lost.

obtained :

$$
\left(\begin{array}{c}
M(t) \\
S(t) \\
I(t) \\
R(t)
\end{array}\right)=\exp (t A)\left(\begin{array}{c}
M(0) \\
S(0) \\
I(0) \\
R(0)
\end{array}\right)
$$

For the initial states, we assume that $M(0)+I(0)+S(0)+R(0)=1$ and that there are no animals with acquired immunity, i.e. $R(0)=0$. The exponential of a matrix as given in equation (5) is computed using the function expm of the statistical software R. The model may be simplified in order to test different hypotheses and to reduce the number of parameters. Model 21 postulates that the infection rates for animals with maternal antibodies and for animals with acquired immunity are equal. Thus the

$$
\left(\begin{array}{c}
d M(t) / d t \\
d S(t) / d t \\
d I(t) / d t \\
d R(t) / d t
\end{array}\right)=\left[\begin{array}{cccc}
-(a+f) & 0 & 0 & 0 \\
a & -\beta & 0 & d \\
f & \beta & -\mu & e \\
0 & 0 & \mu & -(e+d)
\end{array}\right] *\left(\begin{array}{c}
M(t) \\
S(t) \\
I(t) \\
R(t)
\end{array}\right)=A^{*}\left(\begin{array}{c}
M(t) \\
S(t) \\
I(t) \\
R(t)
\end{array}\right)
$$

where $A$ is shorthand for the matrix of transition rates. Here, $M(t)$ is the juvenile part of the proportion of animals with IFAT $+/ \mathrm{PCR}-$ at age $t, S(t)$ the proportion of animals with IFAT $-/ \mathrm{PCR}-$ at age $t, I(t)$ the proportion of animals with IFAT \pm / $\mathrm{PCR}+$ at age $t$ and $R(t)$ the remaining adult proportion of animals with IFAT $+/ \mathrm{PCR}-$ at age $t$. The system of ODEs can be solved by integrating the left and the right term in equation (4) over the time range $[0, t]$ and the following explicit solution is parameter $f$ is set equal to $e$ in the ODE's. Model 22 postulates that passive maternal immunity has no effect on the infection rate and $f$ is set equal to $\beta$. In model 23 it is assumed that the presence of antibodies does not affect the infection pressure at all, so that all 3 parameters $e, f$ and $\beta$ are equal. The symbols and parameters as well as the model descriptions are summarized in Tables 2 and 3.

The transmission matrix (4) and matrix exponentiation (5) allow an intuitive extension of model 
Table 2. Symbols and biological interpretation of the parameters

\begin{tabular}{ll}
\hline \hline Symbol & Biological interpretation \\
\hline$\alpha$ & Rate of loss of maternal antibodies \\
$\beta$ & Infection rate of adult susceptible animals $\left(S_{2}\right)$ \\
$f$ & Infection rate of animals with colostral antibodies \\
$g$ & Infection rate of young susceptible animals $\left(S_{1}\right)$ \\
$k$ & Transition rate from $S_{1}$ to $S_{2}$ \\
$\mu$ & Parasite elimination rate \\
$e$ & (Re-)infection rate of immune animals \\
$d$ & Antibody elimination rate \\
$M(0)$ & Initial proportion of animals in the cohort \\
$S(0)$ & with colostral antibodies \\
$S_{1}(0)$ & Initial proportion of susceptible animals in the cohort \\
$S_{2}(0)$ & Initial proportion of young susceptible animals in the cohort \\
$I(0)$ & Initial proportion of adult susceptible animals in the cohort $(=0)$ \\
$R(0)$ & Initial proportion of infected animals in the cohort \\
\hline \hline
\end{tabular}

Table 3. Notation and description of models used

Notation Model description

Two-compartment models

10 Two-compartment model considering susceptible $(S)$ and infected $(I)$ animals. Animals are infected with a rate $\beta$ and lose infection with a rate $\mu$ (equation (2)).

Four-compartment models

$20 \quad$ Four-compartment model considering animals with colostral antibodies $(M)$, susceptible $(S)$, infected $(I)$ and animals with acquired immunity $(R)$. Animals lose colostral antibodies with a rate $a$. $M$ and $S$ become infected with rates $f$ and $\beta$ respectively. Infection is lost with a rate $\mu$ and acquired antibodies are lost at a rate $d$. Animals with acquired immunity become reinfected at a rate $e$ (equation (5)).

21 As 20, but infection rates for animals with colostral antibodies and animals with acquired immunity are equal: $f=e$.

22 As 20, but colostral antibodies are postulated to have no effect on the infection rate: $f=\beta$

23 As 20, but presence of any antibodies is assumed not to affect the infection rate: $\beta=e=f$.

23* $\quad$ Model used to compute the likelihood of model 10 to compare it to four and five compartment models. Similar to model 23, but with fixed values for the parameters $\beta, \mu$ and $I(0)$ estimated using model 10.

Five-compartment models

30 As 20 but additionally considering different infection rates for neonate susceptible $\left(S_{1}\right)$ and adult susceptible animals $\left(S_{2}\right)$. $S_{1}$ are infected with the rate $g$ and transfer to $S_{2}$ with a rate $k . S_{2}$ are infected with the rate $\beta$ (equation (6)).

31 As 30 , but the infection rate for neonates with colostral antibodies and without colostral antibodies are assumed to be equal $f=g$.

32 As 30 , but colostral antibodies are considered protective for infections $f=0$.

33 As 30, but adult animals are considered protected against disease $\beta=0$.

Subdivions by sex ${ }^{\mathrm{a}}$

20A Model 20 applied to the total sample.

20B Model 20 applied to females, males and geldings separately.

20C Model 20 applied to \{females and males combined and \{geldings\} separately.

20D Model 20 applied to \{females and geldings combined and $\{$ males\} separately.

20E Model 20 applied to \{females\} separately and \{males and geldings combined\}.

a Notation analogous for models 21-33. 
10 to incorporate immunological information. Medley et al. (1993) have developed similar models for theileriosis using ODEs, but the number of parameters to be estimated in their models was very high. Because no adequate samples were available to estimate all parameters simultaneously, they estimated some parameters based on single ODEs and the corresponding parts of the data. This procedure introduces bias into the estimates (Randolph and Nuttall, 1994). Even if the present model oversimplifies the complexity of the actual disease processes, it allows one to simultaneously estimate all parameters based on the same data set, and thus to incorporate interaction effects between PCR and IFAT information. This finally provides a more accurate description of the transmission dynamics.

\section{Five-compartment model}

The assumption for the four-compartment model that the infection rates are age-independent may not be adequate. Thus the model is further expanded by subdividing the compartment of susceptibles into young susceptible animals $\left(S_{1}\right)$ and old susceptible animals $\left(S_{2}\right)$ as depicted in Fig. 3. The principal dynamics of the model are the same as in the fourcompartment model, however $S_{1}$ become infected at a rate $g$ different to the rate $\beta$ at which adult susceptible animals $\left(S_{2}\right)$ are infected. $S_{1}$ transfer to $S_{2}$ with a rate $k$. The corresponding system of ODEs in matrix form is

$\begin{array}{crr}0 & 0 & 0 \\ -(k+g) & 0 & 0 \\ k & -\beta & 0 \\ g & \beta & -\mu \\ 0 & 0 & \mu\end{array}$

The symbols and parameters are summarized in Table 2. In analogy to the four-compartment model, the system of ODEs is solved by integrating the left and the right terms in equation (6) over the time range $[0, t]$ to obtain the explicit solution. For the initial states, we assume that $M(0)+I(0)+S_{1}(0)+$ $S_{2}(0)+R(0)=1$ and that $R(0)=S_{2}(0)=0$. The procedures in the statistical software $\mathrm{R}$ are identical to those utilized for the four-compartment model. Again, variants of the model allow the testing of particular hypotheses. Model 31 assumes that animals with colostral antibodies and young susceptible animals have the same rate of infection $(f=g)$. Model 32 assumes that foals with colostral antibodies are protected from infection $(f=0)$ and model 33 assumes that adult susceptible animals are immune to infection $(\beta=0)$. A summary of the model variants is presented in Table 3 .

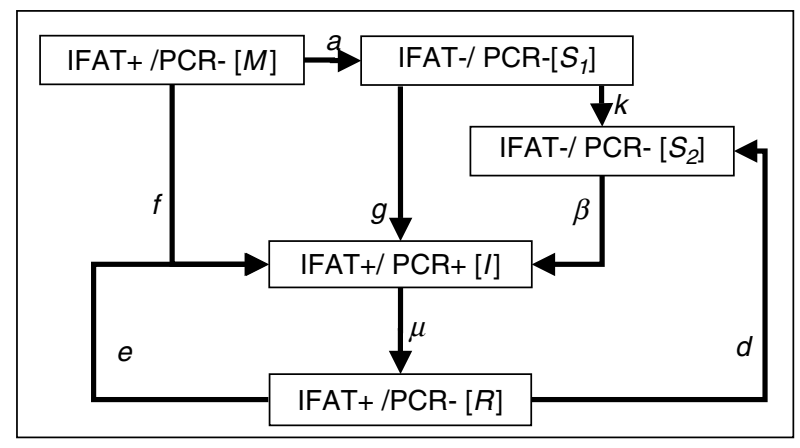

Fig. 3. Graphical representation of model 30. Five compartments represent the maternally protected subpopulation $(M)$, the populations of susceptible foals $\left(S_{1}\right)$, susceptible adults $\left(S_{2}\right)$, infected $(I)$ and immune $(R)$ animals. At the end of each age-interval animals move between the compartments with age-independent rates: The rates $f, g, \beta$ and $e$ correspond respectively to the acquisition rates for foals with passive maternal protection, for foals without humoral protection, for adult animals without humoral protection and animals with acquired immunity. The parasites are eliminated with rate $\mu$, and $a$ and $d$ are the respective rates at which maternal and acquired antibodies are lost. Susceptible foals transfer to the compartment of susceptible adults at a rate $k$.

\section{Maximum likelihood estimation}

Model 10 returns a proportion of infected animals $I(t)$ as a function of age $t$. This corresponds to the probability of an animal being infected at age $t$. The proportion of susceptible horses at age $t$ corresponds

$$
\left.\begin{array}{c}
0 \\
0 \\
d \\
e \\
-(e+d)
\end{array}\right] *\left(\begin{array}{c}
M(t) \\
S_{1}(t) \\
S_{2}(t) \\
I(t) \\
R(t)
\end{array}\right)=A^{*}\left(\begin{array}{c}
M(t) \\
S_{1}(t) \\
S_{2}(t) \\
I(t) \\
R(t)
\end{array}\right) .
$$

to $1-I(t)$. Assuming that the infection statuses of the horses in the sample are independent of each other, the estimation of the parameters is based on the maximization of the following binomial likelihood function $(L)$ :

$L(\beta, \mu, I(0))=\prod_{i=1}^{N} I\left(t_{i}\right)^{x_{i}}\left(1-I\left(t_{i}\right)\right)^{1-x_{i}}$,

where $N=$ number of individuals in the population; $x_{i}=$ infection status ( 1 or 0 ) of individual $i$ (PCR); $t_{i}=$ age of individual;

or equivalently, the maximization of the following log-likelihood function $(L L)$ :

$L L(\beta, \mu, I(0))=\sum_{i=1}^{N}\left\{x_{i} \log \left(I\left(t_{i}\right)\right)+\left(1-x_{i}\right) \log \left(1-I\left(t_{i}\right)\right)\right\}$. 
Models 20, 21, 22 and 23 (Table 3) consider 4 compartments based on the combinations of 2 conditions (PCR and IFAT, compare Table 1) and return the proportion of animals in each compartment at any given age $t$. The probability of finding an animal in a given compartment thus corresponds to this proportion at the corresponding time $t$. Assuming independence of the infection and immunological statuses, the likelihood for this case is a multinomial likelihood function:

$$
\begin{aligned}
& L(\beta, \mu, e, f, d, M(0), I(0)) \\
& =\prod_{i=1}^{N} p_{s}\left(t_{i}\right)^{\left(\mathbf{1}-x_{i}\right)\left(\mathbf{1}-y_{i}\right)} p_{I}\left(t_{i}\right)^{x_{i}}\left(p_{M}\left(t_{i}\right)+p_{R}\left(t_{i}\right)\right)^{\left(\mathbf{1}-x_{i}\right) y_{i}} .
\end{aligned}
$$

The corresponding log-likelihood function is :

$$
\begin{aligned}
& \operatorname{LL}(\beta, \mu, e, f, d, M(0), I(0)) \\
& =\sum_{i=1}^{N}\left\{\left(1-x_{i}\right)\left(1-y_{i}\right) \log \left(p_{s}\left(t_{i}\right)\right)+x_{i} \log \left(p_{I}\left(t_{i}\right)\right)\right. \\
& \left.\quad+\left(1-x_{i}\right) y_{i} \log \left(p_{M}\left(t_{i}\right)+p_{R}\left(t_{i}\right)\right)\right\},
\end{aligned}
$$

where $N=$ number of animals in the population; $x_{i}=$ PCR status ( 1 or 0 ) of individual $i$; $y_{i}=$ IFAT status $(1$ or 0$)$ of individual $i$.

Models 30, 31, 32 and 33 (Table 3) consider 5 compartments based on the combinations of 2 conditions (PCR and IFAT, compare Table 1) and return the proportion of animals in each compartment at any given age $t$. Again, assuming independence of the infection and immunological statuses, the likelihood for this case is the following function:

$$
\begin{aligned}
& L(\beta, \mu, e, f, d, M(0), I(0)) \\
& =\prod_{i=1}^{N}\left(p_{s 1}\left(t_{i}\right)+p_{s 2}\left(t_{i}\right)\right)^{\left(1-x_{i}\right)\left(1-y_{i}\right)} p_{I}\left(t_{i}\right)^{x_{i}}\left(p_{M}\left(t_{i}\right)\right. \\
& \left.\quad+p_{R}\left(t_{i}\right)\right)^{\left(1-x_{i}\right) y_{i}} .
\end{aligned}
$$

The corresponding log-likelihood function is :

$$
\begin{aligned}
& \operatorname{LL}(\beta, \mu, e, f, d, M(0), I(0)) \\
& =\sum_{i=1}^{N}\left\{\left(1-x_{i}\right)\left(1-y_{i}\right) \log \left(p_{s 1}\left(t_{i}\right)+p_{S 2}\left(t_{i}\right)\right)\right. \\
& \left.\quad+x_{i} \log \left(p_{i}\left(t_{i}\right)\right)+\left(1-x_{i}\right) y_{i} \log \left(p_{M}\left(t_{i}\right)+p_{R}\left(t_{i}\right)\right)\right\} .
\end{aligned}
$$

The data used to calculate the log-likelihoods (8), (10) and (12) are from the cross-sectional study in southwest Mongolia (Rüegg et al. 2007), where the data sets for T. equi and B. caballi were obtained from the same horse population. To find the maximum likelihood (ML) estimates of the parameters, the $L L$-functions (8), (10) and (12) given the data are maximized using the optim function of the statistical package $\mathrm{R}$. The function is specified to use the ' $\mathrm{L}$ BFGS-B' algorithm (Byrd et al. 1995), a Newton procedure that allows for box constraints. Newton procedures generally provide good local convergence criteria, but since we search for global maxima, the starting points need to be selected carefully. The value of $0 \cdot 1$ for all parameters was assessed to be an appropriate starting point. For nested models, the ML estimates of the embedded model were used as starting point. For biological reasons, the parameter values are constrained to be larger than 0 and $M(0)$ and $I(0)$ are additionally constrained to be smaller than 1 .

To evaluate the effect of gender on disease transmission, the horse population is subdivided into 4 combinations of females, males and geldings and the models are fitted to each of the subpopulation (represented as \{\} ) separately. A model fitted to the whole sample, i.e. $\{$ females, males, geldings\}, is denoted by A (e.g. model 20A). A model fitted to $\{\mathrm{fe}-$ males $\}$, \{males\} and \{geldings separately is referred to as B (e.g. model 20B), a model with a separate fit for the 2 subpopulations \{females + males $\}$ and \{geldings $\}$ is referred as $\mathrm{C}$, for $\{$ females + geldings and $\{$ males $\}$ as $\mathrm{D}$ and finally for $\mathrm{E}$ \{females $\}$ and $\{$ males + geldings (see Table 3 ). The $L L$ value for a particular combination is defined as the sum of the $L L$ s of the individual fits to each of the corresponding subpopulations. Because geldings are castrated at age 1, the starting values of the model for geldings are chosen as the corresponding proportions of $M, S$ (resp. $S_{1}$, and $S_{2}$ for the 5 compartment models), $I$ and $R$ of 1 -year-old \{males\} in the combination $B$ or analogously of 1-year-old $\{\mathrm{fe}-$ males + males $\}$ in the combination C. For simplicity, the general model is referred to without an alphabetical extension (e.g. model 10, model 20, model 21 etc.). The alphabetical suffix is only used for models fitted to a particular subdivision into gender groups.

\section{Model comparison}

Model 10 assumes that antibodies do not influence the infection rate. Because its likelihood function does not take into account the IFAT results, the $L L$ can not be directly compared to those of the 4- and 5compartment models (Table 4). Model 23 is based on the same assumption as model 10 , but includes the IFAT data in calculating the likelihood function. To compare the results from model 10 with the ones of the more complex models, a modified version of model 23 is used. The parameters $\beta, \mu$ and $I(0)$ in model 23 are fixed by using the corresponding ML estimates from model 10 , and the remaining parameters $a, d$ and $M(0)$ are estimated. We will refer to this derived model as model $23 *$. To verify that the remaining parameters $a, d$ and $M(0)$ in model 23* do not have any influence on the PCR prevalence, and 
Table 4. Results of the pair-wise model comparisons using Monte Carlo simulations

\begin{tabular}{|c|c|c|c|c|c|c|c|}
\hline Model I & Model II & $N L L \mathrm{I}$ & NLL II & $\begin{array}{l}\text { Difference } \\
\text { of } N L L\end{array}$ & $\begin{array}{l}\text { Difference in \# } \\
\text { of parameters }\end{array}$ & $\begin{array}{l}\text { Empirical } \\
95 \% \text {-quantile }\end{array}$ & $\begin{array}{l}\text { Empirical } \\
p \text {-value }\end{array}$ \\
\hline \multicolumn{8}{|c|}{ Theileria equi } \\
\hline $23 * \mathrm{~A}$ & $23 \mathrm{~A}$ & $289 \cdot 3$ & $281 \cdot 4$ & $7 \cdot 9$ & 3 & $40 \cdot 70$ & $0 \cdot 149$ \\
\hline $23 \mathrm{~A}$ & $23 \mathrm{C}$ & $281 \cdot 7$ & $271 \cdot 8$ & $9 \cdot 8$ & 4 & $11 \cdot 25$ & $0 \cdot 122$ \\
\hline \multicolumn{8}{|c|}{ Babesia caballi } \\
\hline $23 * \mathrm{~A}$ & $23 \mathrm{~A}$ & $479 \cdot 2$ & $474 \cdot 0$ & $5 \cdot 2$ & 3 & $0 \cdot 85$ & $0 \cdot 004$ \\
\hline $23 \mathrm{~A}$ & $22 \mathrm{~A}$ & $474 \cdot 0$ & $460 \cdot 6$ & $13 \cdot 4$ & 1 & $2 \cdot 38$ & $0 \cdot 002$ \\
\hline $22 \mathrm{~A}$ & $20 \mathrm{~A}$ & $460 \cdot 6$ & $456 \cdot 4$ & $4 \cdot 2$ & 1 & 1.92 & $0 \cdot 008$ \\
\hline $20 \mathrm{~A}$ & $32 \mathrm{~A}$ & $456 \cdot 4$ & $454 \cdot 6$ & $1 \cdot 8$ & 1 & $1 \cdot 51$ & 0.007 \\
\hline $32 \mathrm{~A}$ & $20 \mathrm{D}$ & $454 \cdot 6$ & $446 \cdot 3$ & $8 \cdot 3$ & 6 & $14 \cdot 190$ & $0 \cdot 220$ \\
\hline
\end{tabular}
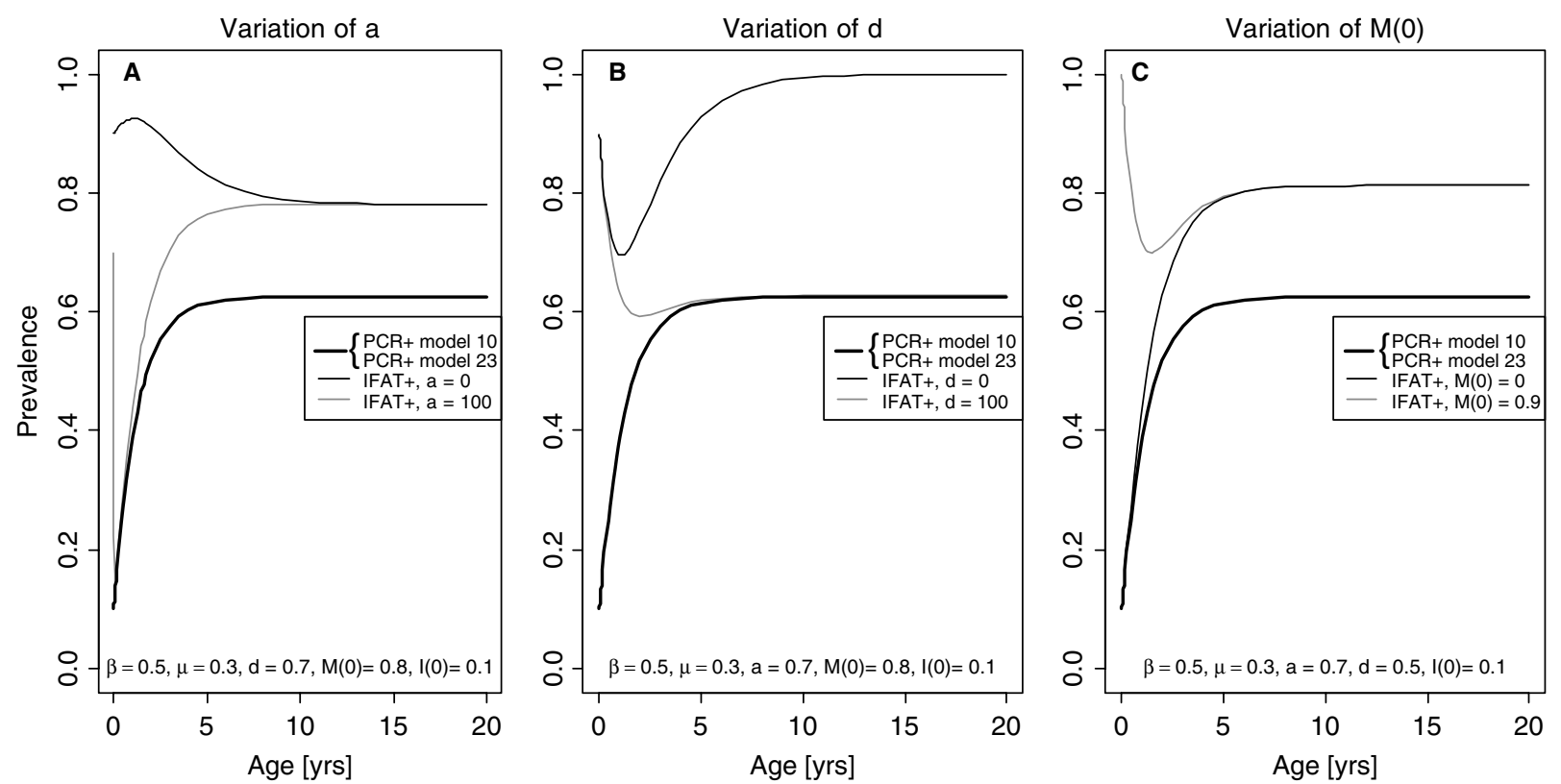

Fig. 4. Effect of varying the model parameters $a$ (panel A), $d$ (panel B) and $M(0)$ (panel C) in model 23 for fixed values of the remaining parameters. The values of the parameters $\beta, \mu$ and $I(\theta)$ are set identical in model 10 and model 23 . To evaluate the effect of varying $a, d$ and $M(0)$, the age-dependent prevalence of PCR + animals is plotted for both models (thick lines) and the proportion of IFAT + animals is plotted for model 23 (thin lines). The parameters $a, d$ and $M(0)$ appear not to have any effect on the PCR prevalence, as the age-dependent prevalences in models 10 and 23 are identical in the graph (thick lines).

hence that model $23 *$ would correctly reflect the PCR prevalence estimated for model 10 , the parameters $a$ and $d$ are varied between 0 and 100 and $M(0)$ is varied between 0 and $1-I(0)$ ( since $M(0)+S(0)+I(0)=1$ ) and the results are compared (Fig. 4$)$. Thus model $23 *$ provides an interface model to compare model 10 to the four- and five-compartment models.

\section{Model selection}

To decide which model performs best with the T.equi and the B. caballi data sets, we first compute the loglikelihood of each of the competing models, and plot them against the number of parameters (Fig. 5). For clarity, the negative log-likelihood scores $(N L L)$ are plotted. Starting with the simplest model $23^{*}$, the models with the steepest decrease in NLL score per number of parameters are further investigated (Fig. 5, grey line). Neighbouring models on this line are compared pair-wise as follows. The difference in NLL of 2 competing models is tested by comparing it with an empirical $95 \%$-quantile. The null hypothesis is that the more complex model does not improve the fit. To accept the more complex model, the reduction of the NLL compared to the simpler model needs to be larger than the empirical $95 \%$-quantile $(\alpha=0 \cdot 05)$, i.e. a reduction of the magnitude observed would occur due to chance alone in less than $5 \%$ of the cases. To compute the empirical $95 \%$-quantile, 500 populations are simulated under the null hypothesis that the simpler model is true. The simulated populations have the same size $(N=510)$ and the same gender 

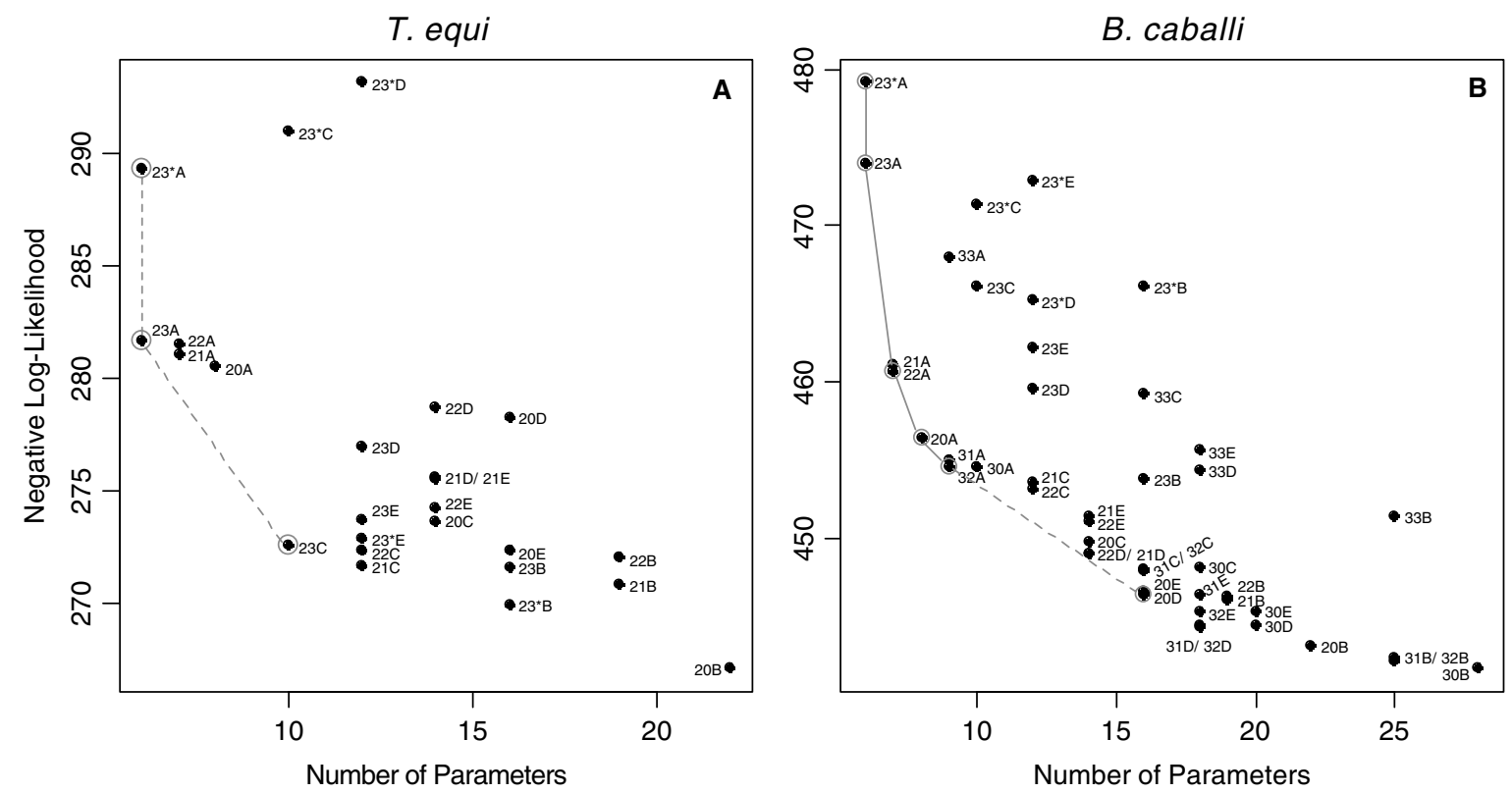

Fig. 5. Comparison of the fitting characteristics of competing models (Table 3) for (A) Theileria equi and (B) Babesia caballi. The negative log-likelihood is plotted against the number of parameters used in the model. The best fitting models are compared pair-wise along the border of the convex hull from the plotted points since these models are better than all competing models with the same number of parameters. Starting with the model with the least number of parameters (grey circles), models on the border are pair-wise compared (grey lines) until a non-significant difference is reached (grey broken line).

distribution ( $\mathrm{f}: \mathrm{m}: \mathrm{g}: \mathrm{NA}=259: 118: 128: 5$ ) as the original data set. A PCR and IFAT status is attributed to the individuals, based on simulation using the simpler model with the ML estimates from the field data. The simulated data sets are then fitted using the same optimization routine as described above, and the difference of the NLL values is calculated for each simulated population. If the optimization does not converge with the default tolerance, the $N L L$-function is scaled by a factor of 10 thereby reducing the tolerance by this factor. Finally, if convergence is still not obtained, the corresponding population is replaced. The $95 \%$-quantile of the resulting empirical sampling distribution of the $N L L$-differences is then compared to the NLLdifference derived from the field data. The pairwise comparisons are continued following the model series represented in Fig. 5 (grey line) until the null hypothesis of no difference between 2 successive models cannot be rejected. The last significant model is considered to best fit the data. For the final selected model, the $95 \%$ bootstrap confidence intervals (CI) of its parameters are computed.

\section{RESULTS}

\section{Models 10 and 23}

If the maximum likelihood estimates for $\beta, \mu$ and $I(\theta)$ of model 10 are introduced into model 23 , the course of the PCR prevalences are identical for both models (Fig. 4). Modification of the parameters $a, d$, and
$M(0)$ in model 23 does not alter the course of the PCR prevalence but has significant impact on the IFAT prevalence.

\section{Best-fitting models}

The minimal values of the negative log-likelihood for all models are plotted versus the number of parameters in Fig. 5. Based on this plot, for T. equi, model $23^{*}$ applied to the undivided population (referred to as $23 * \mathrm{~A}$ ) is compared to model $23 \mathrm{~A}$ and the null hypothesis cannot be rejected at a $5 \%$ test level (empirical $p$-value $=0 \cdot 15$ ). Also the succeeding comparison between model 23A for the undivided population and model $23 \mathrm{C}$ applied to the population subdivided into \{females + males $\}$ and \{geldings revealed no significant difference (empirical $p$ value $=0 \cdot 122$, Fig. 5A, Table 4). These findings indicate that the IFAT does not provide additional significant information to the transmission dynamics process and thus antibodies do not appear to influence the transmission of $T$. equi. The ML estimates for $\beta, \mu$ and $I(0)$ and their $95 \%$ bootstrap confidence intervals are given in Table 5 and the age-dependent PCR-prevalence of the best fitting model is plotted in Fig. 6A. In summary, as $\mu$ is $0 \cdot 014$, T. equi remains as a life-long infection $(95 \%$ CI of $1 / \mu=17 \cdot 9$ years-Infinity). From the ML estimate of $\beta(0 \cdot 446)$ it can be deduced that half the animals are expected to be infected with T. equi at about 2 years of age $(95 \%$ CI of $1 / \beta=1 \cdot 4-3 \cdot 1$ years) and $I(0)$ suggests that 
Table 5. Maximum likelihood estimates (MLE) and $95 \%$ bootstrap confidence intervals (using 500 bootstrapped populations) of the best performing models for Theileria equi and Babesia caballi

\begin{tabular}{lll}
\hline \hline & MLE & $95 \% \mathrm{CI}$ \\
\hline Model 10A for $T$. equi & \\
$\beta$ & $0 \cdot 446$ & $0 \cdot 321-0 \cdot 695$ \\
$\mu$ & $0 \cdot 014$ & $0 \cdot 000-0 \cdot 057$ \\
$I(0)$ & $0 \cdot 125$ & $0 \cdot 043-0 \cdot 207$ \\
Model 32 A for & B. caballi & \\
$a$ & $4 \cdot 249$ & $1 \cdot 566-8 \cdot 370$ \\
$\beta$ & $0 \cdot 054$ & $0 \cdot 010-0 \cdot 305$ \\
$\mu$ & $0 \cdot 653$ & $0 \cdot 196-1 \cdot 097$ \\
$e$ & $0 \cdot 070$ & $0 \cdot 001-0 \cdot 200$ \\
$f$ & - & - \\
$g$ & $1 \cdot 578$ & $0 \cdot 884-7 \cdot 798$ \\
$k$ & $0 \cdot 969$ & $0 \cdot 397-6 \cdot 507$ \\
$d$ & $0 \cdot 00$ & $0 \cdot 00-0 \cdot 158$ \\
$M(0)$ & $0 \cdot 585$ & $0 \cdot 369-0 \cdot 848$ \\
$I(0)$ & $0 \cdot 126$ & $0 \cdot 024-0 \cdot 217$ \\
$S_{1}(0)$ & $0 \cdot 289$ & $0 \cdot 061-0 \cdot 527$ \\
\hline \hline
\end{tabular}

$12 \cdot 5 \%(95 \% \mathrm{CI}: 4 \cdot 3-20 \cdot 7 \%)$ of the population are already infected at birth.

For B. caballi, the models $23 * \mathrm{~A}, 23 \mathrm{~A}, 22 \mathrm{~A}, 20 \mathrm{~A}$, $32 \mathrm{~A}$ applied to the undivided population and model $20 \mathrm{D}$ applied to the population subdivided into $\{\mathrm{fe}-$ males + geldings $\}$ and \{males $\}$ are compared pairwise (grey line, Fig. 5B, Table 4). The first 4 comparisons all reject the null hypothesis that the simpler model performs better (Table 4). The succeeding comparison of model $32 \mathrm{~A}$ to model $20 \mathrm{D}$, however, detects no statistically significant difference (empirical $p$-value $=0 \cdot 220$ ). Thus, a single fit of model 32A for the whole sample represents the epidemiology of $B$. caballi in the sampled population best. A summary of the ML estimates for the parameters and their $95 \%$ bootstrap confidence intervals are given in Table 5. The age-dependent PCR- and IFAT-prevalences of the best fitting model are plotted in Fig. 6B. Thus, colostral antibodies protect foals from infection $(f=0)$ and have a half-life of approximately 3 months $(95 \%$ CI of $1 / a=1 \cdot 4-7 \cdot 6$ months). Further, the infection rates of susceptible foals $(g=1.578)$ differs significantly from that of older susceptible animals $(\beta=0 \cdot 054)$ with susceptible foals being expected to become infected within 8 months (95\% CI of $1 / g=1 \cdot 5$ months $-1 \cdot 2$ years) whereas for adult susceptible animals this is expected after roughly 18 years $(95 \%$ CI of $1 / \beta=3 \cdot 3-100$ years). The rate $k=0.969$ indicates that the susceptible foals are expected to transfer to the category $S_{2}$ at the age of approximately 1 year $(95 \%$ CI of $1 /$ $k=1.8$ months -2.6 years $)$. As $\mu=0.653, B$. caballi is expected to persist in its host for roughly 1.5 years ( $95 \%$ CI of $1 / \mu=10.9$ months $-5 \cdot 1$ years). If an animal has eliminated the parasite, the typical time to acquire a new infection is of the order of 14 years
(95\% CI of $1 / e=5-1000$ years). Thus, with a life expectancy of 20 years re-infection is rather unlikely to occur. The ML estimates for $I(0)$ and $M(0)$ indicate that $\hat{I}(0)=12 \cdot 6 \%(95 \% \mathrm{CI}=2 \cdot 4-21 \cdot 7 \%)$ of foals are infected at birth or very shortly afterwards and $\hat{M}(0)=58 \cdot 5 \%(95 \% \mathrm{CI}=36 \cdot 9-84 \cdot 8 \%)$ receive colostral antibodies from their mothers. Therefore for $100 \%-\hat{I}(0)-\hat{M}(0)=28 \cdot 9 \% \quad(95 \% \quad$ CI $=6 \cdot 0-52 \cdot 7 \%)$ of the births, the mare was not exposed to B. caballi prior to birth. Because the estimate for $d=0 \cdot 00$, antibodies against B. caballi appear not to be eliminated.

\section{DISCUSSION}

The results of the model selection show that $T$. equi and $B$. caballi have very different transmission dynamics, and provide a further piece of evidence for the current debate on the systematic classification of T. equi. The PCR-prevalence of T. equi observed in Fig. 6A shows a cumulative age-dependent course, whereas the prevalence peak at 11 months in Fig. 6B (B. caballi) is very similar to the patterns observed with $B$. bovis and B. bigemina in cattle (Mahoney, 1962). The results of the present study also support the anecdotal reports of various authors (Hourrigan and Knowles, 1979; Schein, 1988; de Waal and van Heerden, 1994) that T. equi remains as a life-long infection, whereas the expected persistence of B. caballi in its host is 1.5 years which is similar to the postulated persistence of 1 to 4 years. The estimated half-life of colostral antibodies against $B$. caballi of 3 months agrees with previous findings that maternal antibody titres against $B$. caballi are already below the detectable cut-off at approximately 4 months of age (Donnelly et al. 1982; Rüegg et al. 2006). The result that acquired antibodies are not eliminated is in agreement with experiments conducted by Tenter (1984), in which IFAT antibodies against $T$. equi and $B$. caballi remained throughout the observations (476 and 190 days post-infection, respectively). Our analysis thus indicates that the approach of estimating host infection rates based on serological data applied by Mahoney and coworkers (Mahoney, 1969; Mahoney and Ross, 1972) is adequate, and may also be applied to equine piroplasmoses. It also implies that for the diagnosis of $T$. equi-positive IFAT results generally correspond to an actual infected status, whereas, for B. caballi, a horse may be seropositive without harbouring the parasite.

In this work, the infection rates $\beta, f, g$ and $e$ are considered to be age independent. The better performance of model 32A compared to model 20A, however, indicates that a differentiation of susceptible foals and susceptible adult animals provides a better fit to the data. Also, the binomial likelihood function assuming independence of the PCR and the IFAT result within the same individual may not be 

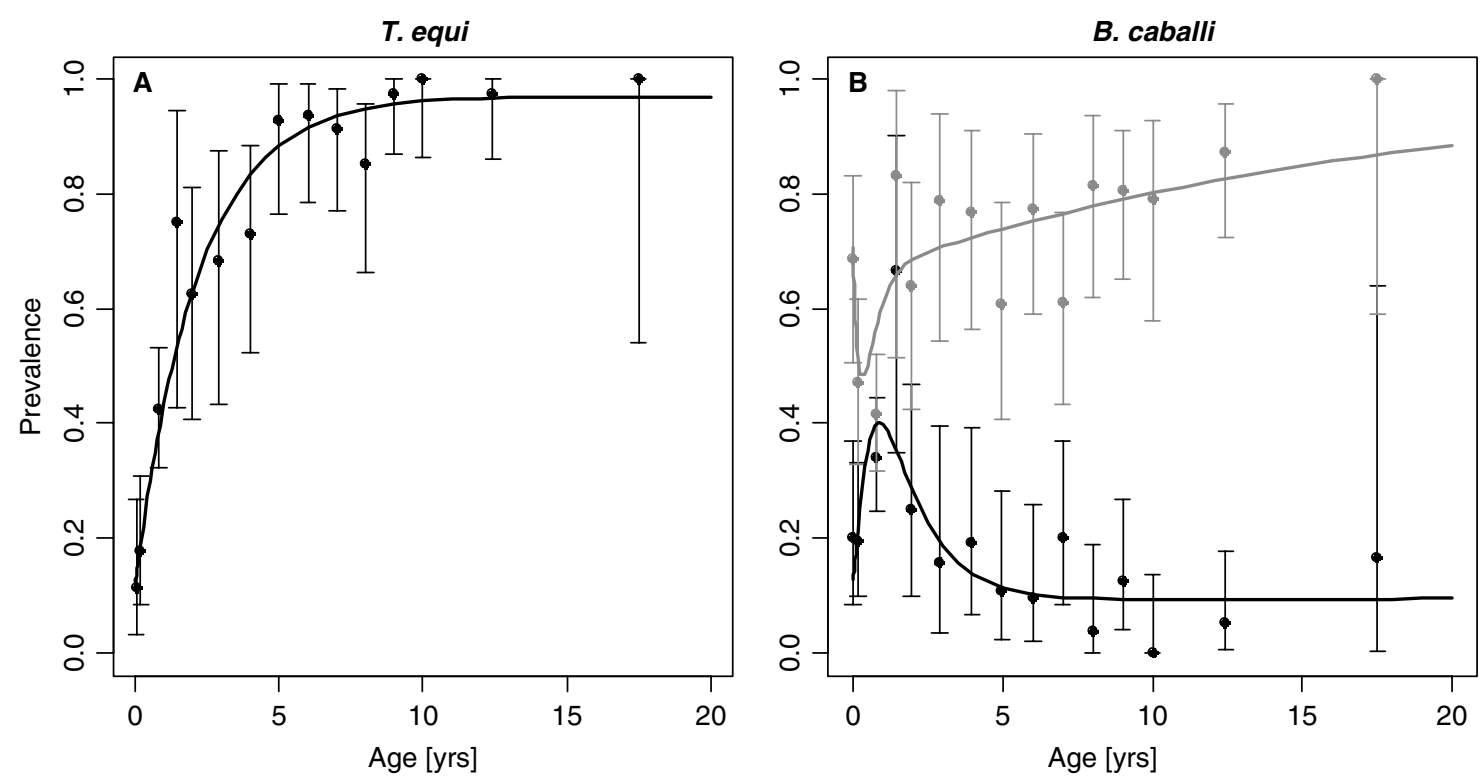

Fig. 6. Plot of the observed PCR (black bullets) and IFAT prevalence (grey bullets) with $95 \%$ binomial confidence intervals (whiskers) for (A) Theileria equi and (B) Babesia caballi. The best fitting models using the maximum likelihood estimates for the corresponding parameters, 10A and 32A respectively, return the age-dependent PCR prevalence (black line) and IFAT prevalence (grey line).

the best choice. More complex models could account for these shortcomings. However, it should be noted that the interpretability of a model suffers with increasing complexity. The exponential dynamics and binomial likelihood of the models presented here can be understood intuitively. It is also the case that the infection rates vary within a year due to varying tick activity. In our model the seasonal variation is averaged to yield simple infection rates per annum. Similarly, the time for interstadial development of the transmitting tick and the resulting latencies in the transmission process are also neglected. The model fit illustrated in Fig. 6 suggests that these simplifications are nonetheless reasonable. It is also not known if the infection rate in ticks remains constant from year to year, and the age dependency that we have found could have been due to increased tick infectivity in the sampling year. However, from anecdotal reports from the owners of the horses investigated, there has not been an increase in piroplasmosis cases in the sampled population, which, considering the difference between $\beta=0.054$ and $g=1 \cdot 578$, would have been expected to be dramatic. It should also be noted that despite confirmed presence of $T$. equi and $B$. caballi in the tick Dermacentor nuttalli (Battsetseg et al. 2002), the observed prevalences in ticks from the study area were $0 \%$ ( $95 \% \mathrm{CI}=0-4 \%$ ) for $T$. equi and $6 \%$ o $(1-17 \%)$ for B. caballi (Rüegg et al. 2007). In the absence of reliable data for the parasite distribution in ticks it is difficult to test additional hypotheses, including the population dynamics of the vector.

The fundamental aim of the approach presented here is to address the shortcomings of the statistical methods often used in epidemiology, such as (a) fitting models with no (meaningful) relationship to the underlying transmission process and (b) making asymptotical assumptions about the test statistics which may not be justified. The approach in the present article provides alternatives to (a) and (b). As an example for (a), methods like generalized linear models (GLM) allow a flexible fit to data, but the resulting parameter estimates are often difficult or even impossible to interpret in biological terms. To address this point, the models presented in this article describe the transmission dynamics of $T$. equi and $B$. caballi with biologically interpretable parameters. Consequently, the parameter estimates can be compared to results from experimental studies to evaluate their reliability and validity. These comparisons have shown that our models provide relevant insights into the epidemiological processes involved in the transmission of equine piroplasmoses. To address point (b), likelihood ratio tests are applied using empirical probability distributions of the test statistic generated with Monte Carlo simulations. Conventionally, a $\chi^{2}$-distribution is used for the likelihood test statistic to select the best fitting model. However, this asymptotical distribution is only valid under conditions which are not satisfied for many of our model comparisons. Indeed, a likelihood ratio test based on a $\chi^{2}$-distribution with 3 degrees of freedom would yield a $p$-value of $0 \cdot 001$ for the comparison of model 23*A and 23A for T. equi as opposed to the non-significant empirical $p$-value of $0 \cdot 122$ based on our Monte Carlo approach. Similarly, for the comparison of model 32A versus $20 \mathrm{D}$ for $B$. caballi, the $p$-value based on a $\chi^{2}$-distribution with 
6 degrees of freedom would be $0 \cdot 011$ compared to the empirical $p$-value of $0 \cdot 221$ which is non-significant. Thus, in each case, the putative significant $p$-values of the test with a $\chi^{2}$-distribution would lead to a wrong model selection.

The authors wish particularly to thank Jean-Pierre Gabriel and Christian Mazza from the Institute of Mathematics at the University of Fribourg and Richard Baltensperger from the College of Engineering and Architecture of Fribourg for the discussion on the solution of the ODEs and the 'Informatikdienste' of the University of Zürich for installing $\mathrm{R}$ on the 'Matterhorn-cluster' so rapidly. We would also like to thank the referees for their extremely valuable comments on the manuscript. This project was partially financed by the Forschungskredit der Universität Zürich 2003.

\section{REFERENCES}

Avarzed, A., de Waal, D. T., Igarashi, I., Saito, A., Oyamada, T., Toyoda, Y. and Suzuki, N. (1997). Prevalence of equine piroplasmosis in Central Mongolia. Onderstepoort Fournal of Veterinary Research 64, 141-145.

Battsetseg, B., Lucero, S., Xuan, X., Claveria, F., Byambaa, B., Battur, B., Boldbaatar, D., Batsukh, Z., Khaliunaa, T., Battsetseg, G., Igarashi, I., Nagasawa, H. and Fujisaki, K. (2002). Detection of equine Babesia spp. gene fragments in Dermacentor nuttalli Olenev 1929 infesting Mongolian horses, and their amplification in egg and larval progenies. Fournal of Veterinary Medical Science 64, 727-730.

Bruning, A. (1996). Equine piroplasmosis an update on diagnosis, treatment and prevention. British Veterinary Fournal 152, 139-151.

Byrd, R. H., Lu, P., Nocedal, J. and Zhu, C. (1995). A limited memory algorithm for bound constrained optimization. SIAM Fournal on Scientific Computing 16, 1190-1208.

Dallwitz, M. J., Young, A. S., Mahoney, D. F. and Sutherst, R. W. (1987). Comparative epidemiology of tick-borne diseases of cattle with emphasis on modelling. International Fournal for Parasitology 17, 629-637.

de Waal, D. T. and van Heerden, J. (1994). Equine babesiosis. In Infectious Diseases of Livestock with Special Reference to South Africa (ed. Coetzer, J., Thomson, G. R. and Tustin, R. C.), pp. 295-304. Oxford University Press, Cape Town.

Donnelly, J., Phipps, L. P. and Watkins, K. L. (1982). Evidence of maternal antibodies to Babesia equi and B. caballi in foals of seropositive mares. Equine Veterinary Fournal 14, 126-128.

Friedhoff, K. T. (1988). Transmission of Babesia. In Babesiosis of Domestic Animals and Man (ed. Ristic, M.), pp. 23-52. CRC Press, Boca Raton (FL), USA.
Gummow, B., de Wet, C. S. and de Waal, D. T. (1996). A sero-epidemiological survey of equine piroplasmosis in the northern and eastern Cape Provinces of South Africa. Fournal of the South African Veterinary Association 67, 204-208.

Heuchert, C. M., de Giulli, V. J., de Athaide, D. F., Böse, R. and Friedhoff, K. T. (1999).

Seroepidemiologic studies on Babesia equi and Babesia caballi infections in Brazil. Veterinary Parasitology 85, 1-11.

Hourrigan, J. L. and Knowles, R. C. (1979). Equine piroplamsosis (E.P.). American Association of Equine Practicioners Nezwsletter 1, 119-128.

Mahoney, D. F. (1962). Epidemiology of babesiosis in cattle. Australian Fournal of Science 24, 310-313.

Mahoney, D. F. (1969). Bovine babesiosis: A study of factors concerned in transmission. Annals of Tropical Medicine and Parasitology 6, 1-14.

Mahoney, D. F. and Ross, D. R. (1972). Epizootiological factors in the control of bovine babesiosis. Australian Veterinary Fournal 48, 292-298.

Medley, G. F., Perry, B. D. and Young, A. S. (1993). Preliminary analysis of the transmission dynamics of Theileria parva in eastern Africa. Parasitology 106, 251-264.

Randolph, S. E. and Nuttall, P. A. (1994). Nearly right or precisely wrong? Natural versus laboratory studies of vector-borne diseases. Parasitology Today 10, 458-462.

Ross, D. R. and Mahoney, D. F. (1974). Bovine babesiasis: computer simulation of Babesia argentina parasite rates in Bos taurus cattle. Annals of Tropical Medicine and Parasitology 68, 385-392.

Rüegg, S. R., Torgerson, P. R., Deplazes, P. and Mathis, A. (2007). Age-dependent dynamics of Theileria equi and Babesia caballi infections in southwest Mongolia based on IFAT and/or PCR prevalence data from domestic horses and ticks. Parasitology 134, 939-947.

Rüegg, S. R., Torgerson, P. R., Doherr, M., Deplazes, P., Böse, R., Robert, N. and Walzer, C. (2006). Equine piroplasmoses at the reintroduction site of the Przewalski's horse (Equus ferus przewalskii) in Mongolia. Fournal of Wildlife Diseases 42, 518-526.

Schein, E. (1988). Equine babesiosis. In Babesiosis of Domestic Animals and Man (ed. Ristic, M.), pp. 197-208. CRC Press, Inc., Boca Raton (FL), USA.

Smith, R. D. (1983). Babesia bovis: computer simulation of the relationship between the tick vector, parasite, and bovine host. Experimental Parasitology 56, 27-40.

Tenter, A. M. (1984). Serodiagnose experimenteller und natuerlicher Piroplasmeninfektionen der Pferde. Doctoral thesis, Tierärztliche Hochschule, Hanover, Germany. 